\title{
Supratotal Resection of Glioblastoma Allows Better Survival Outcomes Than Gross Total Resection
}

\author{
Seung Hyun Baik \\ Kwandong University \\ So Yeon Kim \\ Kwandong University \\ Young Chul Na \\ Kwandong University \\ Jin Mo Cho ( $\nabla$ ns9@naver.com ) \\ Kwandong University
}

\section{Research Article}

Keywords: Glioblastoma, Lobectomy, Gross total resection, Overall survival, Supratotal resection.

Posted Date: September 28th, 2021

DOI: https://doi.org/10.21203/rs.3.rs-597119/v2

License: (9) This work is licensed under a Creative Commons Attribution 4.0 International License. Read Full License 


\section{Abstract}

Objective: Supra-total resection (SupTR) of glioblastoma allows superior long-term disease control and increases overall survival. On the other hand, aggressive conventional approaches, including gross total resections (GTR), are limited by the impairment risk of adjacent eloquent areas, which may cause severe postoperative functional morbidity. This study aimed to analyze institutional cases with respect to the potential survival benefits of additional resection, including lobectomy, as a paradigm for SupTR in patients of glioblastoma.

Methods: Between 2014 and 2018, 15 patients with glioblastoma underwent GTR and additional lobectomy at our institution. The postoperative Karnofsky performance score (KPS), progression-free survival (PFS), and overall survival (OS) were analyzed for the patients.

Results: Patients with SupTR showed significantly prolonged PFS and OS. The median PFS and OS values for the entire study group were 33.5 months ( $95 \%$ confidence intervals [Cl]: 18.5-57.3 months) and 49.1 months (95\% Cl: 24.7-86.6 months), respectively. Multivariate analysis revealed that 06-DNAmethylguanine methyltransferase (MGMT) promoter methylation status was the only predictor for both superior PFS ( $p=0.03$, OR 5.7, 95\% Cl 1.0-49.8) and OS ( $p=0.04,0 R 6.5,95 \% \mathrm{Cl} 1.1-40.2)$. There was no significant difference between the pre- and post-operative KPS scores.

Conclusions: Our results strongly suggest that SupTR with lobectomy allows superior PFS and OS without negatively affecting the patient performance.

\section{Introduction}

Glioblastoma (GBM) is the most common malignant brain tumor. The best treatment option for GBMs is extensive resection followed by chemo-radiotherapy.[1] The extent of resection is the only modifiable prognostic factor associated with good prognosis and increased survival. A previous study reported that the median overall survival (OS) of patients with no residual tumors on postoperative magnetic resonance imaging (MRI, 15.2 months) was significantly longer than the OS in patients with residual tumors.[2]

In neurosurgery, radical resection is not considered a routine procedure, given that wide radical resection causes neurological deficits resulting from resecting normal brain tissue. In 1928, Walter Dandy reported five cases of hemispherectomy for removing gliomas in the right hemisphere.[3] After removing the whole right hemisphere, the patients showed no neurologic deficits apart from hemiplegia; however, there was no improvement in survival rates. One of the patients survived for 3.5 years; however, all the patients died soon. After this report, removing normal brain tissue was considered unsuitable and many neurosurgeons have focused on removing obvious brain tumors visible on MRI while preserving neurological function.

Gross-total resection (GTR) is determined based on the postoperative T1-enhanced MRI. Yan Michael Li reported that the removal of lesions with high signal intensity on T2-FLAIR MRI improves survival.[2] 
However, the cancer cells of a GBM directly invade areas that appear normal on MR images. [4, 5] Therefore, improvement of the survival rate after removing normal brain tissue around the tumor remains unclear.

Hugues Duffau performed supratotal resection (SupTR), which involved extensive resection of the surrounding brain tissue, as well as the lesion, which showed promising results in patients with low-grade gliomas.[6] In case the GBM is located in the less eloquent area, much of the region can be extensively resected. Theoretically, additional resection of non-functioning brain tissue would result in few or no functional deficits.

We previously used to remove enhancing lesions only in GBM occurring in the frontal, temporal, or occipital lobe. We have since adopted the principle of SupTR for GBMs involving a wide resection, including lobectomy, of GBMs. This study aimed to review the survival and performance outcomes in patients with GBM.

\section{Methods}

\section{Patient Selection}

This retrospective study was approved by our institutional review board, which waived the requirement for patient informed consent given the retrospective nature of this study. All procedures were in accordance with the ethical standards of the institutional and/or national research committee and the 1964 Declaration of Helsinki and its later amendments or comparable ethical standards.

Between Feb 2014 and Jan 2018, 41 patients who were newly diagnosed with GBM and underwent surgery at our institute involving complete tumor resection in the frontal, temporal, or occipital lobe. We excluded patients with tumors involving eloquent areas.

SupTR was defined as complete resection determined by a lack of visible residual tumors on the T1enhanced postoperative MRI and additional frontal-temporal or occipital lobectomy. Two neuroradiologists separately reviewed the MRI scans to determine successful GTR in each patient. The general performance status of each patient was evaluated using Karnofsky Performance Status (KPS) scores, which were recorded preoperatively and at 4 postoperative weeks. All the patients received postoperative concomitant chemo-radiotherapy and adjuvant chemotherapy with temozolomide, as previously described.[1] We excluded patients with a visible residual tumor on postoperative MRI scans.

\section{Lobectomy Procedure}

After tumor removal, the anterior pole of the frontal lobe was removed under navigation guidance. Next, a subpial dissection was performed at the medial, lateral, and inferior surfaces of the frontal lobe, while preserving the surrounding vascular structures and olfactory nerve. The posterior margin of the frontal lobectomy was just beneath the coronal suture, which is approximately $1-2 \mathrm{~cm}$ anterior to the precentral sulcus. An awake craniotomy was performed in cases when the tumor was near an eloquent area. 
Moreover, tumor removal and/or additional anterior temporal lobectomies for cases with temporal lobe tumors. The posterior resection margin was approximately $5-6 \mathrm{~cm}$ from the temporal pole. When the tumor was located in the occipital lobe, the whole occipital lobe was resected regardless of visual symptoms. Figure 1 illustrates some representative cases.

\section{Molecular Diagnostics}

Genetic information regarding the tumor was retrospectively collected from electronic medical records. We examined 06-DNA-methylguanine methyltransferase (MGMT) promoter methylation status using a methylation-specific polymerase chain reaction, as previously described. Isocitrate dehydrogenase mutations were detected through direct sequencing or immunohistochemistry using the R132H mouse monoclonal antibody.

\section{Statistical Analysis}

The OS was analyzed using the Kaplan Meier method. The Wilcoxon signed-rank test was used to compare pre- and postoperative KPS scores. All statistical analyses were performed using IBM SPSS software version 24.0 (IBM Corp.). Statistical significance was set at a p-value $<0.05$.

\section{Ethical Committee}

This retrospective study was approved by Catholic kwandong Univeristy Institutional review board, which also waived the requirement for patient informed consent given the retrospective nature of this study

\section{Results}

\section{Patient Characteristics}

During the study period, 41 patients underwent surgery for GBM at our institution. Among them, 15 patients had GBMs confined in the frontal, temporal, or occipital lobes; further, they had undergone complete tumor resection. Table 1 summarizes the characteristics of all the patients. The median age was 64 years (range 29-79 years). Among the patients, 9 (60.0\%) were male and 8 (53.3\%) harbored a methylated MGMT promoter. The median preoperative and postoperative KPS scores were 90 (range 40100 ) and 80 (range 40-100), respectively. In 14 patients, 5-ALA fluorescence guidance was used for surgery. There was no significant difference between the pre- and postoperative KPS scores $(p=0.356$, related-samples Wilcoxon signed-rank test) 
Table 1

Patient characteristics

\begin{tabular}{|ll|}
\hline No of patient & 15 \\
\hline Age at operation & $64(29-79)$ \\
\hline Female sex & $9(60.0 \%)$ \\
\hline KPS score pre-OP & $75(60-100)$ \\
\hline KPS score post-OP & $80(60-100)$ \\
\hline Frotal / Temporal / Occipital & $8 / 4 / 3$ \\
\hline 5-ALA fluorescence & 14 used / 1 unused \\
\hline IDH mutant / wild type & $6(40 \%) / 9(60 \%)$ \\
\hline MGMT methlylated / Unmethylated & $8(53.3 \%) / 7(46.7 \%)$ \\
\hline
\end{tabular}

The median follow-up duration was 29.8 months $(95 \% \mathrm{Cl}$ 23.5-86.6 months, reverse Kaplan-Meier method). (Fig. 2) At the last observation time, 3 (20.0\%) patients were dead and 12 (80.0\%) patients were alive. The median PFS and OS values were 33.5 months ( $95 \% \mathrm{Cl} 26.5-57.3$ months) and 49.1 months (95\% Cl 24.7-86.6 months), respectively.

We performed multivariate logistic progression analysis with the following variables: age, preoperative KPS score, postoperative KPS score, and MGMT methylation status. This analysis revealed that MGMT methylation status was the only significant and independent predictor for both prolonged PFS $(p=0.03$, OR 5.7, 95\% Cl 1.0-49.8) and prolonged OS ( $p=0.04$, OR 6.5, 95\% Cl 1.1-40.2). (Table 2)

Table 2

Multivariate analysis of survival

\begin{tabular}{|lllllll|}
\hline & \multicolumn{7}{l}{ PFS } & \multicolumn{7}{l|}{ OS } \\
\cline { 2 - 6 } & Adjusted OR & $\mathbf{9 5 \%} \mathrm{Cl}$ & $\mathbf{p ~ v a l u e}$ & Adjusted OR & $\mathbf{9 5 \%} \mathrm{Cl}$ & p value \\
\hline Age & 0.8 & $0.9-1.0$ & 0.7 & 0.9 & $0.8-1.1$ & 0.8 \\
\hline MGMT & 5.7 & $1.0-49.8$ & 0.03 & 6.5 & $1.1-40.2$ & 0.04 \\
\hline Pre-OP KPS score & 1.2 & $1.1-39.4$ & 1 & 1.3 & $0.1-2.4$ & 0.9 \\
\hline Post-OP KPS score & 1.1 & $0.2-2.4$ & 0.8 & 1 & $0.9-1.5$ & 0.9 \\
\hline
\end{tabular}

\section{Discussion}

Many studies have reported that the extent of resection is a crucial positive prognostic factor in patients with GBM [7-10] The extent of the resection results is positively correlated with the progression-free and overall survival.[9] However, there can be tumor recurrence even after GTR and standard chemo- 
radiotherapy. The extent of resection is defined based on postoperative T1-weighted contrast-enhanced MRI results. Nevertheless, there can be infiltration of GBM tumor cells well beyond contrast-enhancing areas. $[11,12]$ Even when postoperative MRI images show removal of enhancing lesions, there can be tumor recurrence due to infiltrating tumor cells. [5] Most recurrent tumors occur adjacent to the resection margin or within $2 \mathrm{~cm} .[4,13,14]$

This problem can be solved with the use of 5-ALA, which allows broader tumor resection since it reveals infiltrating tumor cells beyond the enhancing region.[15] Although GTR was performed based on postoperative MRI, patients with residual 5-ALA fluorescent tumors at the surgical field showed worse outcomes than those without residual fluorescence.[16] However, in this study with a small sample size, the use of 5-ALA did not significantly affect outcomes.

Given the recent emergence of SupTR, the exact interpretation of SupTR remains unclear.[17-19] Duffau used this term to describe extended resection with a margin beyond the MRI-defined abnormalities in lowgrade gliomas [18]. Therefore, we used T2 and FLAIR images as references for SupTR in low-grade gliomas. For GBMs, the term SupTR has been used to describe resection beyond the contrast-enhancing lesion.[20]

This study considered lobectomy as part of the SupTR procedure. This procedure resulted in a significantly larger volume of the resection cavity compared with the initial tumor volume. Therefore, a larger resection cavity volume than the initial tumor volume can be considered as a criterion for SupTR.

In our study, the median PFS and median OS were 33.5 and 49.1 months, while the respective values in conventional GTR are 12 and 16 months.[20] These findings indicated that SupTR procedures can provide clinical benefits for carefully selected patients with GBM showing non-eloquent tumor localization. This study proposes excision of the entire lobectomy as a paradigm for SupTR. Therefore, these findings are suggestive of lobectomy as an aggressive SupTR policy that constitutes the surgical modality of choice. However, lobectomy as an oncosurgical resection tool bears the risk of a postoperative decline in language, memory, and visual loss. However, given our retrospective design, a similar analysis was beyond the feasibility of our study. Subsequent prospective study designs might allow assessment of neurocognitive issues, and therefore provide a more comprehensive view of lobectomy as a potential seminal oncosurgical therapeutic strategy for GBM.

This study has several limitations. The main limitation was the acquisition of retrospectively collected data. Specifically, the patients were not randomized and treated according to the physician's decision. However, to rule out the heterogeneity of non-eloquent tumor localization, we applied highly selective inclusion criteria for GBMs located in the frontal, temporal, and occipital lobes, which resulted in a small sample size. Additionally, the sample size was too small to reach a conclusion. There is a need for other large-scale studies. Finally, we only included data from a single center.

\section{Conclusions}


Lobectomy significantly improves postoperative functional status, as well as PFS and OS, in patients with GBM. Our findings demonstrate that GTR plus additional lobectomy can improve PFS and OS without functional deterioration. Therefore, lobectomy might be the surgical modality of choice as an aggressive SupraTR policy for GBM. The extent of resection is positively correlated with the improvements in outcomes. Since the extent of resection is the only modifiable prognostic factor in patients with GBM, this surgical strategy could improve their outcomes.

\section{Abbreviations}

GBM: glioblastoma

GTR: gross total resection

KPS: Karnofsky performance score

MGMT: 06-DNA-methylguanine methyltransferase

MRI: magnetic resonance imaging

OS: overall survival

PFS: progression-free survival

SupTR: supra-total resection

\section{Declarations}

Compliance with Ethical Standards

* Disclosure of potential conflicts of interest : The authors declare that they have no conflict of interest

* Research involving human participants and/or animals

This article does not contain any studies with human participants or animals performed by any of the authors.

* Funding

This research did not receive any specific grant from funding agencies in the public, commercial, or notfor-profit sectors.

\section{Data availability}


The datasets generated during and/or analysed during the current study are available from the corresponding author on reasonable request.

\section{Author Contribution}

All authors (Seung Hyun Baik M.D, So Yeon Kim M.D., Young Chul Na M.D., Jin Mo Cho, M.D., Ph.D) contribute eqully to this artifle.

\section{References}

1. Stupp, R. et al. Radiotherapy plus concomitant and adjuvant temozolomide for glioblastoma. New England journal of medicine, 352, 987-996 (2005).

2. Li, Y. M., Suki, D., Hess, K. \& Sawaya, R. The influence of maximum safe resection of glioblastoma on survival in 1229 patients: can we do better than gross-total resection? Journal of neurosurgery, 124, 977-988 (2016).

3. Dandy, W. E. Removal of right cerebral hemisphere for certain tumors with hemiplegia: Preliminary report. Journal of the American Medical Association, 90, 823-825 (1928).

4. Minniti, G. et al. Patterns of failure and comparison of different target volume delineations in patients with glioblastoma treated with conformal radiotherapy plus concomitant and adjuvant temozolomide. Radiotherapy and Oncology, 97, 377-381 (2010).

5. Yamahara, T. et al. Morphological and flow cytometric analysis of cell infiltration in glioblastoma: a comparison of autopsy brain and neuroimaging. Brain tumor pathology, 27, 81-87 (2010).

6. Duffau, H. Long-term outcomes after supratotal resection of diffuse low-grade gliomas: a consecutive series with 11-year follow-up. Acta neurochirurgica, 158, 51-58 (2016).

7. D’Amico, R. S., Englander, Z. K., Canoll, P. \& Bruce, J. N. Extent of resection in glioma-a review of the cutting edge. World neurosurgery, 103, 538-549 (2017).

8. Lacroix, M. et al. A multivariate analysis of 416 patients with glioblastoma multiforme: prognosis, extent of resection, and survival. Journal of neurosurgery, 95, 190-198 (2001).

9. Sanai, N., Polley, M-Y., McDermott, M. W., Parsa, A. T. \& Berger, M. S. An extent of resection threshold for newly diagnosed glioblastomas. Journal of neurosurgery, 115, 3-8 (2011).

10. Sawaya, R. Extent of resection in malignant gliomas: a critical summary. Journal of neuro-oncology, 42, 303 (1999).

11. Nagashima, G. et al. Graphic analysis of microscopic tumor cell infiltration, proliferative potential, and vascular endothelial growth factor expression in an autopsy brain with glioblastoma. Surgical neurology, 51, 292-299 (1999).

12. TAMURA, M., OHYE, C. \& NAKAZATO, Y. Pathological anatomy of autopsy brain with malignant glioma. Neurologia medico-chirurgica, 33, 77-80 (1993). 
13. Konishi, Y., Muragaki, Y., Iseki, H., Mitsuhashi, N. \& Okada, Y. Patterns of intracranial glioblastoma recurrence after aggressive surgical resection and adjuvant management: retrospective analysis of 43 cases. Neurologia medico-chirurgica, 52, 577-586 (2012).

14. Milano, M. T. et al. Patterns and timing of recurrence after temozolomide-based chemoradiation for glioblastoma. International Journal of Radiation Oncology* Biology* Physics, 78, 1147-1155 (2010).

15. Eyüpoglu, I. Y. et al. Supra-complete surgery via dual intraoperative visualization approach (DiVA) prolongs patient survival in glioblastoma. Oncotarget, 7, 25755 (2016).

16. Aldave, G. et al. Prognostic value of residual fluorescent tissue in glioblastoma patients after gross total resection in 5-aminolevulinic acid-guided surgery. Neurosurgery, 72, 915-921 (2013).

17. de Leeuw, C. N. \& Vogelbaum, M. A. Supratotal resection in glioma: a systematic review. Neurooncology, 21, 179-188 (2019).

18. Duffau, $\mathrm{H}$. Is supratotal resection of glioblastoma in noneloquent areas possible? World neurosurgery, 82, e101-e103 (2014).

19. Esquenazi, Y. et al. The survival advantage of "supratotal" resection of glioblastoma using selective cortical mapping and the subpial technique. Neurosurgery, 81, 275-288 (2017).

20. Pessina, F. et al. Maximize surgical resection beyond contrast-enhancing boundaries in newly diagnosed glioblastoma multiforme: is it useful and safe? A single institution retrospective experience. Journal of neuro-oncology, 135, 129-139 (2017).

\section{Figures}




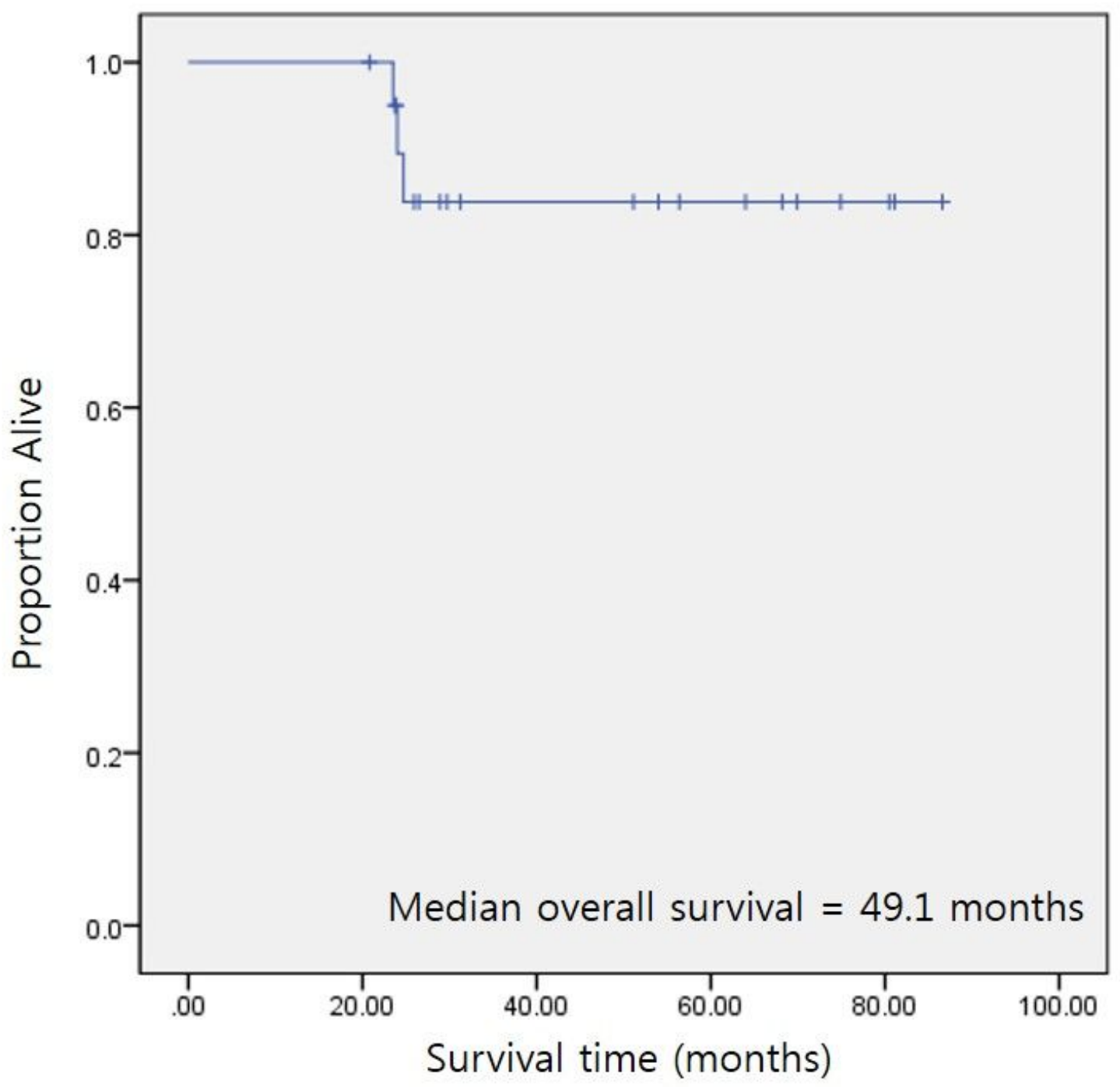

Figure 1

Kaplan-Meier overall survival curve in the study with a newly diagnosed GBM. 
(A)

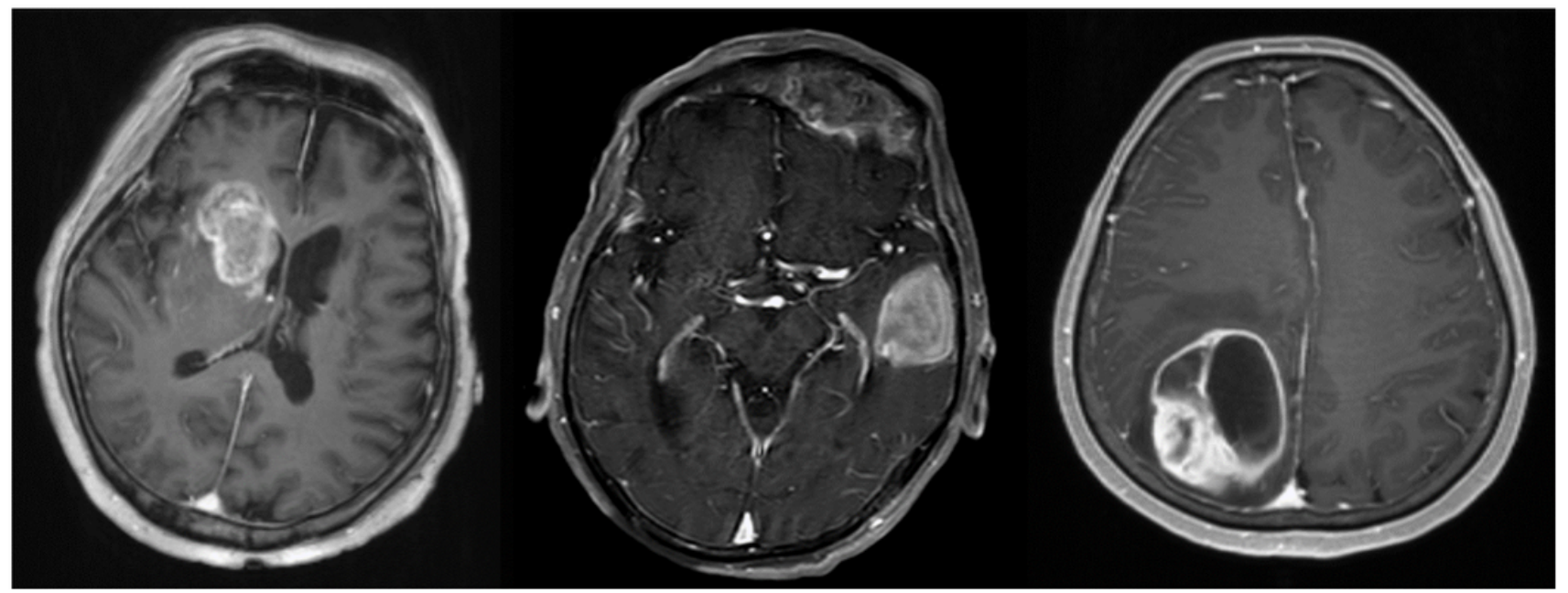

(B)

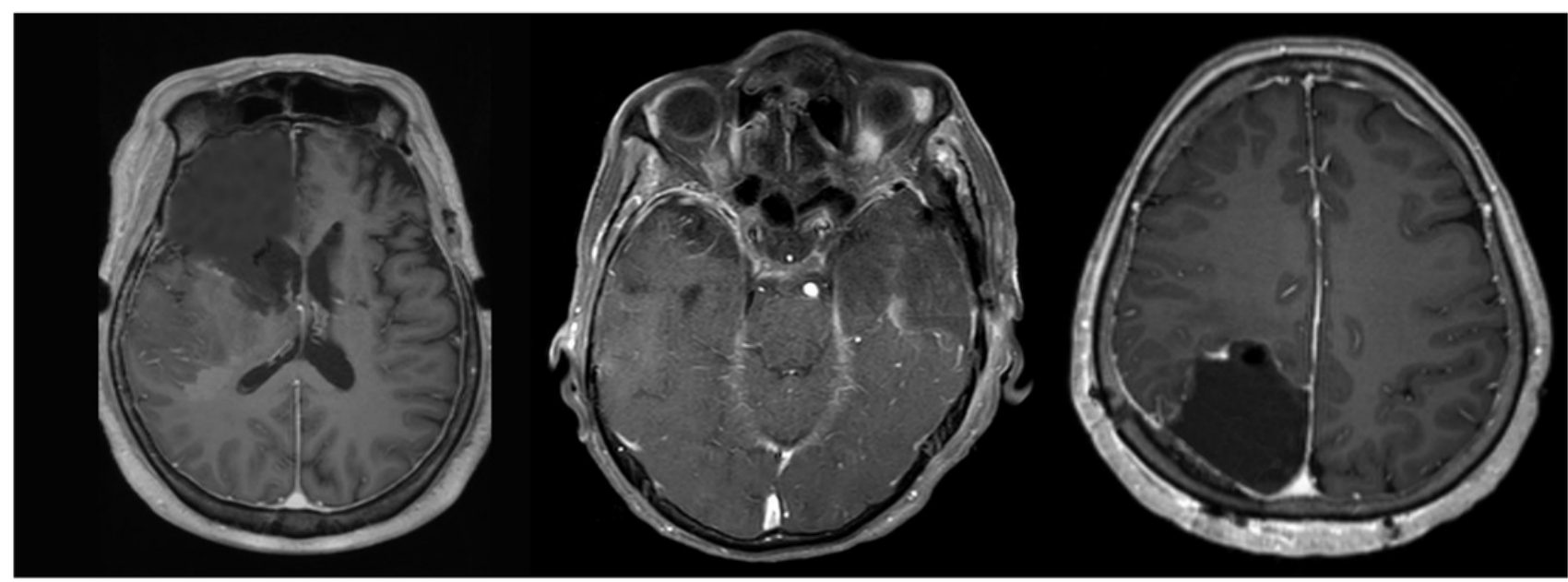

Figure 2

A. Preoperative axial T1-weighted gadolinium-enhanced MRI showing a GBM involving the frontal, temporal and occipital lobe. B. An example of a patient in whom SupTR was achieved. After complete resection of the tumor, a frontal, temporal and occipital lobectomy was performed. 